\title{
Problemática narcísica-identitária em adolescentes abusadores sexuais: contribuições do Rorschach Escola de Paris ${ }^{1}$
}

\author{
Lana dos Santos Wolff, Deise Matos do Amparo² \\ Universidade de Brasília, Brasília-DF, Brasil \\ Roberto Menezes de Oliveira \\ Universidade Católica de Brasília, Brasília-DF, Brasil \\ Jean Yves Chagnon \\ Université Paris 13, Paris, França
}

\section{RESUMO}

Este artigo analisa a problemática narcísica-identitária de adolescentes que cometeram abuso sexual. Parte-se da hipótese de que é diante dos remanejamentos psíquicos ocorridos na adolescência e das dificuldades do aparelho psíquico em exercer a função de paraexcitação que pode ocorrer a passagem ao ato violento. Foi utilizado o Método de Rorschach na perspectiva da abordagem francesa da Escola de Paris, com grupo único de 4 adolescentes, de idades entre 15 e 18 anos, que praticaram violência sexual. A interpretação dos protocolos considerou os investimentos nos limites, as defesas narcísicas e seus efeitos, o investimento na representação de si, a identidade e a identificação. Conclui-se que a dificuldade de definição dos limites, a problemática identificatória, a inibição e o restrito manejo pulsional, bem como o estabelecimento de relações especulares com o uso de defesas narcísicas, indicam falhas no processo de subjetivação.

Palavras-chave: adolescência; abuso sexual; Teste de Rorschach; identidade; narcisismo.

ABSTRACT - Problematic narcissistic-identity in adolescent sex abusers: Contributions from the Parisian Rorschach School This article analyzes the narcissistic-identitary problematic of adolescents who commit sexual abuse. It starts with the hypothesis that in adolescence, a psychic rearrangement occurs and difficulties begin within the psychic apparatus that exercises the stopexcitation function, which may lead to a violent act. We used the Rorschach Method in the French Approach of Parisian School, with a group of four adolescents aged 15 to 18 who had committed acts of sexual violence. The interpretation of the protocols considered the investment in the limits; the narcissistic defense and its effects, investment in the representation of the self, the identity and the identification. The study concluded that the difficulty of defining the limits, identification problematic, inhibition and limited drive management, in addition to the establishment of specular relations using narcissistic defenses indicated flaws in the process of subject choice.

Keywords: adolescence; sexual abuse; Rorschach Test; identity; narcissism.

\section{RESUMEN - Problemática de la identidad-narcisista en adolescentes ofensores sexuales: las contribuciones del Rorschach de la Escuela de Paris}

Este artigo analiza la problemática de la identidad-narcisista de los adolescentes que han cometido abuso sexual. Se basa en la hipótesis de la reubicación psíquica que ocurre en la adolescencia y de las dificultades del aparato psíquico al ejercer la función de para-excitación, pueden llegar a un acto violento. Fue utilizado el Método de Rorschach en la perspectiva del abordaje francés de la Escuela de Paris, con un grupo único de cuatro adolescentes con las edades entre 15 y 18 años, que habían cometido violencia sexual. La interpretación de los protocolos consideró los investimentos en los limites; las defensas narcisistas y sus efectos, el investimento en la auto-representación, la identidad y la identificación. Tras el estudio, se concluye que la dificultad en la delimitación de los limites, la problemática identificación, la inhibición y la restricción del manejo de los impulsos, además del establecimiento de relaciones especulares usando las defensas narcisistas indican fallas en el proceso de subjetivación.

Palabras clave: adolescencia; abuso sexual; Teste de Rorschach; identidad; narcisismo.

${ }^{1}$ Pesquisa realizada com apoio do Conselho Nacional de Desenvolvimento Científico e Tecnológico e da Coordenação de Aperfeiçoamento de Pessoal de Nível Superior (Bolsa de Pós-doutorado Senior).

${ }^{2}$ Endereço para correspondência: Programa de Pós-Graduação em Psicologia Clínica e Cultura, Departamento de Psicologia Clínica, Instituto de Psicologia

Universidade de Brasília, Campus Universitário Darcy Ribeiro, ICC. Ala Sul, Bloco A, Térreo, 70910-900, Brasília-DF, Brasil. E-mail: deise.amparo.matos@gmail.com 
As produções teóricas psicanalíticas clássicas ressaltam as transformações da adolescência no que se refere ao corpo, à identidade e ao psiquismo (Aberastury \& Knobel, 1981/1970; Blos, 1998). Alguns pesquisadores franceses têm discutido os remanejamentos e as modificações desse período, ressaltando o tema do traumatismo e das dimensões narcísicas-identitárias inerentes à adolescência (Chagnon, 2009; Gutton, 1990, 2002; Jeammet \& Corcos, 2005; Marty, 2000). Nesse sentido, Gutton (1990, 2002) problematiza o processo adolescente considerando esse momento como atravessado por uma violência interna de essência pubertária vinculada às transformações corporais e psíquicas da puberdade. O pubertário seria o correspondente psíquico do que acontece no corpo.

Assim, a adolescência é reativada por três eixos organizadores: complexo de Édipo, narcisismo e elaboração da perda de objeto (Emmanuelli \& Azoulay, 2008). Este trabalho teve o objetivo de abordar as questões relativas ao eixo narcísico-identitário, considerando que as demandas edípicas e objetais perpassam e entrelaçam a dimensão abordada. Segundo Jeammet (2006), as bases narcísicas dependem da qualidade dos investimentos objetais; tal raciocínio remete a uma situação paradoxal: "como ser si mesmo se, para sê-lo, é preciso ao mesmo tempo ser como o outro e se diferenciar do outro?" (Jeammet \& Corcos, 2005, p. 56). Essa contradição pode ser compreendida a partir da teoria de Winnicott (1984/2005), que remonta às experiências precoces do lactente e ao cuidado materno.

As bases da constituição narcísica estão vinculadas à qualidade dessas interações precoces e ao processo de investimento do bebê em seu corpo (autoerotismo). Se no decorrer do desenvolvimento a mãe suficientemente boa propiciou a interiorização dos vínculos, não há conflito entre a necessidade da ligação, essa dependência do objeto, e a necessária autonomia (Jeammet, 2005; Winnicott, 1984/2005). De modo geral, são as bases narcísicas que possibilitam o suporte para o sentimento de continuidade e segurança nas relações objetais (Emmanuelli \& Azoulay, 2008; Jeammet \& Corcos, 2005), e a identidade constituída relaciona-se aos processos de diferenciação, individuação e identificação (Rausch de Traubenberg \& Sanglade, 1984).

Segundo a hipótese de Jeammet (2006), é diante dos remanejamentos narcísicos e identitários que ocorrem na adolescência, e das dificuldades do aparelho psíquico em exercer a função de paraexcitação, que pode ocorrer a passagem ao ato violento. Dessa forma, o ato seria uma tentativa de proteção ao narcisismo e à identidade. No entanto, essa defesa utilizada pelo adolescente mostra-se ineficiente ao buscar no mundo perceptivo-motor um controle das tensões e dos conflitos intrapsíquicos. Se em um primeiro momento a passagem ao ato protege o ego, tal movimento impossibilitará o trabalho de ligação entre afeto e representação e implicará em uma falta de investimento egoico.

Marty $(2000,2010,2012)$ também indica a falta de apoio do sistema de paraexcitação para conter e transformar a demanda pulsional como outra hipótese para passagem ao ato agressivo dos adolescentes. $\mathrm{O}$ agir seria uma tentativa de simbolização ao tratar na realidade externa o que não pode ser elaborado internamente, um modo de obter alívio da angústia provocada pela excitação pulsional. Esse autor emprega uma metáfora interessante para a compreensão desse aspecto: o aparelho digestivo da aranha (Marty, 2010). Para se alimentar, o inseto necessita degradar o alimento fora de seu corpo, pois não possui um sistema digestivo que realize esse trabalho. De modo semelhante, nos adolescentes que passam ao ato de forma violenta parece não haver espaço psíquico interno para metabolizar a violência, porque os recursos psíquicos para escoamento pulsional deveriam ter sido desenvolvidos ao longo do período de latência.

Ao abordar a violência sexual praticada por adolescentes, Roman (2012), assim como Balier (1996/2008), que se refere aos adultos, considera que esse comportamento está desvinculado da sexualidade genital, porém encontra-se relacionado a uma problemática primitiva, referente à constituição psíquica e ao processo de subjetivação, com angústias que mobilizam defesas radicais como a clivagem do eu, baseada na recusa da realidade. Roman (2012), em seu trabalho com adolescentes em diversos casos judiciários envolvendo esse tipo de ato, ressalta também a problemática do que denomina o "mesmo", tratando da relação entre autor e vítima vinculada ao registro da igualdade. Esse elemento de paridade pode estar relacionado, muitas vezes, à idade próxima entre o autor e a vítima, ao pertencimento a uma mesma família (biológica ou não) e ao conhecimento, por parte do autor, acerca dos interesses e das atividades da vítima.

No que se refere aos estudos sobre adolescentes em conflito com a lei utilizando o Método de Rorschach, principalmente com o grupo específico de adolescentes que cometeu abuso sexual, observamos número restrito de pesquisas na área. Uma das investigações realizadas examinou 120 adolescentes do sexo masculino, com idades entre 12 e 17 anos, em conflito com a lei e levantou indicadores de validade de critério do Método de Rorschach no Sistema Compreensivo, analisando a diferença entre aqueles que cometeram furtos e aqueles que cometeram homicídios (Durat Júnior, 2006). Os dados desse estudo denotaram que os adolescentes que haviam cometido homicídio tendiam a apresentar hostilidade em relação ao meio, revelando oposição negativa e desconsideração às pessoas, em atitude de autoafirmação com evitação de contatos mais afetivos. Esses adolescentes também não apresentavam sinais de ansiedade e tensão. O grupo que estava sob medida de restrição de liberdade por furto apresentava sinais de ansiedade e de insatisfação consigo mesmo, além de maior motivação para mudanças que o grupo anterior.

Outro estudo na perspectiva da Abordagem do Sistema Compreensivo foi realizado por Souza e Rezende (2012) com dois estudos de caso, um por homicídio e outro por furto. No primeiro caso, o adolescente envolvido com furto apresentou dificuldade em lidar com afetos e tendência a descargas emocionais violentas, com 
distanciamento das relações interpessoais e baixa autoestima, dificuldade com os relacionamentos interpessoais e aspectos depressivos no seu protocolo. Já no caso de homicídio, emergiram traços de constrição afetiva, ausência de ansiedade e remorso, dificuldade de reconhecer e expressar sentimentos, baixa autoestima, autoimagem e percepção do outro baseadas em experiências reais, capacidade de estabelecer relações interpessoais adaptativas.

No que se refere ao sistema francês de abordagem do Método de Rorschach, Amparo e Pereira (2010) apresentaram estudo de caso de um adolescente que estava sob medida de liberdade assistida e havia praticado abuso sexual com uma criança. Neste caso, o protocolo do Rorschach foi marcado por inibição e coartação, com investimento na formalização. No que se refere ao eixo narcísico-identitário, esse adolescente apresentou estabilidade na representação de si, mas a referência ao duplo em espelho nas pranchas II, III e VII evidenciou a conotação narcísica dessa representação. A hipótese levantada pelas autoras é de que a representação de si era constituída em vertente narcísica e a inibição indicava os escassos recursos de mentalização, sendo a passagem ao ato sexual violento uma forma de substituir o trabalho psíquico de ligação.

Em estudo realizado por Roman (2004), na França, com dez adolescentes que praticaram ato sexual violento, os jovens foram divididos em dois grupos distintos. No primeiro deles, verificou-se que, nos protocolos do Rorschach e do Teste de Apercepção Temática (TAT) de 5 sujeitos com idades entre 13 e 14 anos, envolvidos em situações de abuso sexual sem a prática de estupro, havia homogeneidade. As técnicas projetivas apontaram para a instabilidade das bases narcísicas agregada a uma precária vinculação objetal, a qual sinalizava nos testes um ataque à integridade e uma ligação volátil e persecutória com o objeto. Nesse estudo, o autor observou, no primeiro grupo, que a elaboração da posição depressiva estava presa às questões identitárias, com fragilidade identificatória, na qual a diferença de sexos e de gerações era tênue ou inexistente. A representação humana ficava diluída e apagada e, no Rorschach, emergia em respostas como "pessoas", "bonecos de neve". O segundo grupo, de adolescentes entre 14 e 17 anos, com prática de abuso sexual, contrastava na sua constituição. Com três adolescentes o contato foi satisfatório, sob um fundo de sedução; os outros dois se encontravam em uma verdadeira ruptura de relação evocada por um quadro psicótico. Ficou evidente que o processo de adolescer era dominado por movimentos de retirada narcisista e objetal e pela construção de objetos de ligação que reforçavam, de modo frágil, as bases narcísicas e a clivagem. Como ocorreu no primeiro grupo, as identificações eram frágeis e não sustentavam a elaboração do conflito. Essas identificações estavam vinculadas às representações humanas inacessíveis ou unitárias, com ênfase especial no feminino.

Chagnon (2008) discutiu, em seu estudo, o caso de um adolescente ofensor sexual que apresentava identidade pouco constituída, desintegrada e indiferenciada do suporte materno. As respostas ao teste de Rorschach, muitas vezes, apresentavam imagens interpenetradas, confusas, pouco diferenciadas e com referências às origens e ao corpo materno. As identificações efetuavam-se de maneira fálico-feminina, em um modelo de fusão com a imago materna, estando ausente a imagem masculina viril. Apesar da fusão com a figura materna e do contato precário com a realidade, o sujeito (por meio de mecanismos de defesa narcísicos, como estilo rígido, respostas "pele", desdobramento e idealização) realizava tentativas de assegurar sua identidade. Em recente publicação, Pelladeau e Chagnon (2015) apresentaram estudo de caso considerando o processo identitário e identificatório de um adolescente, autor de agressão sexual, a partir da análise de três problemáticas centrais: identitária-narcísica, objetal e nível de elaboração mental.

Esses estudos apresentam enfoques diferentes e, apesar da diversidade de abordagens ou referências normativas no Rorschach, alguns pontos podem ser levantados, como a discussão sobre as fragilidades relativas às dimensões afetivas, relacionais, narcísicas e identitárias da organização psíquica desses adolescentes. Em relação à propriedade do Método de Rorschach para avaliar as bases narcísicas e identitárias, encontramos nas referências da Escola de Paris os indicadores para realizar essa análise (Chabert, 1993, 1998/2003, 2004; Emmanuelli \& Azoulay, 2008).

Segundo Chabert (1998/2003), as diferentes respostas de conteúdo humano indicam a capacidade de identificação da pessoa em relação a uma imagem humana, habilidade que fundamenta a identidade. Os conteúdos humanos podem se manifestar nos protocolos do Rorschach de diferentes maneiras: inteiros $(\mathrm{H})$, parcializados $(\mathrm{Hd})$ ou como personagens místicos, irreais $((\mathrm{H}))$. Nos protocolos, caso as respostas humanas parciais estejam acompanhadas de maior número de respostas humanas inteiras, provavelmente a imagem de si está minimamente integrada. No entanto, se o $\mathrm{H} \%$ - percentual de respostas humanas - for constituído praticamente por conteúdos parcializados, isso pode enunciar uma falha na constituição da imagem do corpo, com a presença de angústias muito primitivas, como a fragmentação (Chabert, 1998/2003). Já as representações humanas ou animais com registro no imaginário (gnomos, anões, fadas, entre outros) indicam afastamento apoiado no imaginário e na intelectualização (Emmanuelli \& Azoulay, 2008).

Em seu aspecto estrutural, as pranchas unitárias e compactas (I, IV, V, VI e IX) possibilitam a projeção de imagens, indicando a representação de si e da identidade. Nessas pranchas, o sujeito será confrontado em sua capacidade de estabelecer limites entre o dentro e o fora, entre o eu e o outro, que poderão indicar uma imagem do corpo diferenciada, integrada e portadora de um espaço psíquico. Por outro lado, podem surgir representações de si desintegradas e fragmentadas, indicando problemática identitária (Chabert, 1993, 1998/2003, 2004). As pranchas bilaterais (II, III e VII) introduzem a especularidade e o conflito em uma dimensão relacional. 
Além dos aspectos indicados, as qualidades cromáticas e sensoriais do Método de Rorschach também suscitam o narcisismo do sujeito. A presença do negro-cinza-branco, da cor vermelha e dos tons pastéis traz à tona, respectivamente, os sentimentos de vazio e as fantasias em relação à morte, a relação do sujeito com seus objetos internos, suas demandas pulsionais, e a relação com os objetos externos (Chabert, 1993). Para que o sujeito tenha a possibilidade de realizar positivo investimento narcísico, é necessário que a capacidade de diferenciar entre o dentro e o fora, entre o eu e o outro, esteja presente, de forma que haja um suporte mínimo para o estabelecimento da identidade (Emmanuelli \& Azoulay, 2008). A qualidade da análise dos investimentos nos limites permite levantar indicadores sobre a problemática narcísica e poderá ser identificada no Rorschach pela análise das respostas globais; pelos determinantes formais e pela maneira como os conteúdos de barreira-penetração surgem no teste (Chabert, 2004, 1993,1998/2003; Emmanuelli \& Azoulay, 2008; Masling, 1999). A análise das defesas, tal como elas se manifestam no Método de Rorschach, como negação, congelamento dos movimentos pulsionais, idealização ou desdobramento, também são indicadores do funcionamento narcísico (Chabert, 1993; Emmanuelli \& Azoulay, 2008).

Outro aspecto que permite analisar a problemática narcísica na relação com a identidade é o investimento na representação de si, sendo este item evidenciado no Rorschach pela análise da prancha $\mathrm{V}$ e pelos conteúdos humanos e animais de todas as pranchas do teste (Chabert, 1993;
Emmanuelli \& Azoulay, 2008; Rausch de Traubenberg, 1970/1998). A análise das respostas de movimentos (K, kan, kob, kp) no Rorschach são especialmente relevantes para análise da representação de si e da identidade.

Em síntese, este estudo considerou a propriedade do Método de Rorschach para análise da problemática narcísica-identitária e pretendeu abordar as especificidades e a dinâmica dessa questão em adolescentes que cometeram violência sexual. $\mathrm{O}$ uso de técnicas projetivas, em uma perspectiva psicodinâmica contribui para a compreensão do funcionamento psíquico, indicando recursos da personalidade e potencialidades para mudanças (Chabert, 2004).

\section{Método}

Este estudo foi fundamentado na proposta metodológica de Husain (1991) de construção de um grupo único, constituído a partir de uma problemática ou sintomatologia específica, buscando as variações e a unidade estrutural interna ao grupo, dispensando o uso de um grupo de controle. Nesse sentido, as técnicas projetivas têm muito a contribuir para a compreensão do diagnóstico e também para realizar uma reflexão sobre as singularidades das diversas organizações de personalidade em semelhantes disposições sintomáticas. Essa dupla polaridade reabilita a prática das pesquisas que têm por base o grupo único.

Participaram desta pesquisa 4 adolescentes com idades entre 15 e 18 anos que praticaram violência sexual. A Tabela 1 apresenta informações que caracterizam a organização familiar, o contexto da passagem ao ato, o tipo

Tabela 1

Informações gerais sobre os adolescentes que participaram da pesquisa

\begin{tabular}{|c|c|c|c|c|c|}
\hline Nome & Idade & Organização familiar & $\begin{array}{l}\text { Contexto da passagem } \\
\text { ao ato sexual violento }\end{array}$ & $\begin{array}{c}\text { Medida } \\
\text { socioeducativa }\end{array}$ & $\begin{array}{l}\text { Contexto da } \\
\text { avaliação }\end{array}$ \\
\hline Cristiano & 15 & $\begin{array}{l}\text { Pai: comerciante; } \\
\text { Mãe: contadora; } \\
\text { Reside com os pais e } \\
\text { um irmão gêmeo. }\end{array}$ & $\begin{array}{l}\text { Sozinho com uma criança de } \\
\text { três anos de idade que morava } \\
\text { em uma residência próxima. } \\
\text { A situação de abuso envolveu a } \\
\text { prática de sexo oral. }\end{array}$ & $\begin{array}{c}\text { A violência sexual } \\
\text { não foi denunciada } \\
\text { à justiça. }\end{array}$ & $\begin{array}{l}\text { Clínica } \\
\text { escola }\end{array}$ \\
\hline Paulo & 17 & $\begin{array}{c}\text { Mãe: professora; } \\
\text { Reside com a mãe, o } \\
\text { padrasto e uma irmã } \\
\text { mais velha. }\end{array}$ & $\begin{array}{l}\text { Sozinho com uma criança na } \\
\text { sua casa. A situação de abuso } \\
\text { envolveu penetração. }\end{array}$ & $\begin{array}{c}\text { Internação durante } \\
30 \text { dias e liberdade } \\
\text { assistida. }\end{array}$ & $\begin{array}{l}\text { Clínica } \\
\text { escola }\end{array}$ \\
\hline Leonardo & 18 & $\begin{array}{c}\text { Pai: pedreiro; } \\
\text { Madrasta: dona de casa; } \\
\text { Reside com os irmãos, } \\
\text { o pai e a madrasta. } \\
\text { Mãe faleceu quando o } \\
\text { adolescente tinha } 16 \text { anos. }\end{array}$ & $\begin{array}{l}\text { Com um grupo de amigos } \\
\text { enquanto estava embriagado. } \\
\text { A situação de abuso ocorreu } \\
\text { na rua e envolveu conjunção } \\
\text { carnal com uma mulher. }\end{array}$ & $\begin{array}{l}\text { Medida privativa } \\
\text { de liberdade. }\end{array}$ & $\begin{array}{l}\text { Unidade de } \\
\text { internação }\end{array}$ \\
\hline Jonas & 16 & $\begin{array}{c}\text { Mãe: padeira } \\
\text { (com histórico de } \\
\text { alcoolismo); } \\
\text { Padrasto: padeiro; } \\
\text { Reside com a mãe, o } \\
\text { padrasto e } 11 \text { irmãos. }\end{array}$ & $\begin{array}{l}\text { Com uma mulher na rua, } \\
\text { acompanhado de um amigo. }\end{array}$ & $\begin{array}{l}\text { Medida privativa } \\
\text { de liberdade. }\end{array}$ & $\begin{array}{l}\text { Unidade de } \\
\text { internação }\end{array}$ \\
\hline
\end{tabular}


de medida socioeducativa e o contexto da realização da avaliação dos adolescentes.

Os participantes foram encaminhados por psicólogos e/ou assistentes sociais que os acompanhavam na unidade de internação e na clínica escola para realização da avaliação psicológica. Os jovens e seus responsáveis assinaram o Termo de Consentimento Livre e Esclarecido (TCLE); logo em seguida, foram realizadas as entrevistas e aplicado o Método de Rorschach. Esta pesquisa foi aprovada por Comitê de Ética em Pesquisa (Protocolo 02-06/2011) e está de acordo com a Resolução no 466/12, do Conselho Nacional de Saúde (CNS). Os nomes utilizados são fictícios, para preservar a identidade dos participantes da pesquisa.

Os protocolos do Método de Rorschach foram aplicados e analisados na perspectiva da Escola de Paris (Azoulay et al., 2007; Chabert, 1993, 1998/2003, 2004; Chagnon, 2013; Emmanuelli \& Azoulay, 2008; Ikiz et al., 2010; Jardim-Maran, Pasian, \& Okino, 2015; Pasian, 2010;
Rausch de Traubenberg, 1970/1998; Verdon \& Amparo, 2013). A classificação das respostas foi realizada por três codificadores independentes, sendo que as discordâncias foram discutidas até se chegar a um consenso final. A interpretação dos protocolos considerou a abordagem psicanalítica do Método de Rorschach, fundamentalmente nos aspectos relativos ao eixo narcísico-identitário (Chabert, 1993, 1998/2003, 2004; Emmanuelli \& Azoulay, 2008; Rausch de Traubenberg, 1970/1998).

\section{Resultados e Discussão}

Em relação ao conjunto dos protocolos dos adolescentes, a Tabela 2 apresenta os principais indicadores encontrados no Rorschach dos adolescentes, segundo a Escola de Paris. A análise de alguns desses indicadores permitiu particularizar a investigação sobre a problemática narcísica-identitária desse grupo.

Tabela 2

Principais indicadores do Rorschach encontrados nos protocolos dos adolescentes na perspectiva da Escola de Paris

\begin{tabular}{|c|c|c|c|c|c|}
\hline Indicadores & Cristiano & Paulo & Leonardo & Jonas & Norma* \\
\hline $\mathrm{R}$ & 32 & 15 & 12 & 28 & 17,70 \\
\hline G\% & 31,25 & 53,33 & 58,33 & 7,14 & 35,00 \\
\hline $\mathrm{D} \%$ & 31,25 & 33,33 & 25,00 & 39,28 & 33,40 \\
\hline Dd\% & 31,25 & 13,33 & 16,66 & 46,42 & 30,30 \\
\hline Dbl\% & 6,25 & 0 & 0 & 7,14 & 1,10 \\
\hline $\mathrm{F} \%$ & 56,25 & 53,33 & 41,66 & 42,85 & 54,50 \\
\hline $\mathrm{F}+\%$ & 66,66 & 37,50 & 100,00 & 50,00 & 55,60 \\
\hline$F+e x t \%$ & 75,00 & 66,66 & 83,33 & 46,42 & 57,30 \\
\hline $\mathrm{H} \%$ & 18,75 & 40,00 & 8,33 & 21,42 & 20,90 \\
\hline $\mathrm{A} \%$ & 46,87 & 46,66 & 58,33 & 42,85 & 51,00 \\
\hline Ban\% & 9,37 & 20,00 & 16,66 & 10,71 & 17,00 \\
\hline $\mathrm{K}: \Sigma \mathrm{k}$ & $1: 7$ & $2: 3$ & $1: 2$ & $0: 1$ & $0,8: 1,7$ \\
\hline $\mathrm{FC}: \mathrm{CF}+\mathrm{C}$ & $1: 0$ & $1: 0$ & $1: 1$ & $6: 3$ & $2,1: 1,9$ \\
\hline$F C^{\prime}: C^{\prime} F+C^{\prime}$ & $2: 0$ & $2: 3$ & $1: 0$ & $2: 0$ & - \\
\hline $\mathrm{FE}: \mathrm{EF}+\mathrm{E}$ & $3: 0$ & $0: 0$ & $1: 0$ & $4: 0$ & $0,4: 0,7$ \\
\hline Controle dos impulsos ( $\Sigma \mathrm{C}: \Sigma \mathrm{E})$ & $0,5: 1.5$ & $0,5: 0$ & $2: 0,5$ & $7: 2$ & - \\
\hline Tipo de ressonância íntima (K: $\Sigma \mathrm{C})$ & Coartativo & $\begin{array}{l}\text { Introversivo } \\
\text { dilatado }\end{array}$ & $\begin{array}{c}\text { Extratensivo } \\
\text { dilatado }\end{array}$ & $\begin{array}{l}\text { Extratensivo } \\
\text { puro }\end{array}$ & \\
\hline 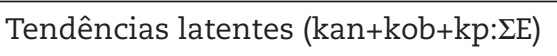 & $7: 1,5$ & $3: 0$ & $2: 0,5$ & $1: 2$ & - \\
\hline $\mathrm{H}+\mathrm{A}: \mathrm{Hd}+\mathrm{Ad}$ & $12: 7$ & $9: 2$ & $8: 0$ & $7: 10$ & - \\
\hline $\mathrm{H}: \mathrm{Hd}$ & $1: 5$ & $3: 1$ & $1: 0$ & $0: 6$ & - \\
\hline Barreira/penetração & $2: 3$ & $2: 2$ & $2: 2$ & $5: 3$ & - \\
\hline Fórmula da angústia** & 18,75 & 26,66 & 0 & 42,85 & - \\
\hline IRA $^{* * *}$ & 31,25 & 40,00 & 25,00 & 32,14 & - \\
\hline
\end{tabular}

Nota: *as normas consideradas para análise dos protocolos foram retiradas de Jardim-Maran (2011) e Jardim-Maran, Pasian e Okino (2015); **Hd+(Hd)+Anat+Sg+Fg+Sex x 100/R; ***IRA=100 X número de respostas VIII+IX+X/R; IRA=Índice de Reatividade Afetiva. 


\section{Os investimentos nos limites e as defesas narcísicas}

O investimento nos limites foi analisado inicialmente pelas respostas globais, já que, segundo Emmanuelli e Azoulay (2008), indicam a capacidade de estabelecimento mínimo de identidade pela apreensão do objeto enquanto inteiro. Paulo e Leonardo apresentaram índices de globalização acima da média (respectivamente, $\mathrm{G} \%=53,33 \%$ e $\mathrm{G} \%=58,33 \%$ ). Em Cristiano e Jonas, as respostas globais ficaram um pouco rebaixadas (respectivamente, $G \%=31,25 \%$ e $G \%=7,14 \%$ ). De todo modo, o número de respostas globais encontra-se alterado em relação ao que é esperado na adolescência, segundo os dados normativos (Jardim-Maran, Pasian, \& Okino, 2015). No que concerne à qualidade das respostas, observou-se a presença de respostas globais simples em todos os protocolos e globais organizadas nos protocolos de Cristiano e Paulo. Especificamente, as respostas globais simples aparecem associadas aos conteúdos híbridos (Cristiano e Jonas); às percepções disfóricas (Cristiano); ao espaço em branco (Leonardo) ou ao fenômeno especial de envolvimento (Jonas e Paulo). Apesar disso, algumas respostas globais simples estão associadas aos determinantes de boa forma, evidenciando seu caráter adaptativo, como é o caso de Paulo, na prancha V, verbalizando "Um morcego" (G F+A), e de Cristiano, na prancha VI, referindo "Uma nave" (G F+Obj.).

Assim como na análise das respostas globais, a apreciação dos determinantes formais (Emmanuelli \& Azoulay, 2008) evidenciou, em Cristiano e Paulo, uma potencialidade no investimento dos limites. No entanto, essa tentativa de delimitação entre interno e externo não se mostra suficientemente eficaz em Paulo ( $\mathrm{F} \%=53,33 \%$; $\mathrm{F}+\%=37,5 \%$ ). Por outro lado, as respostas de forma dos protocolos de Jonas ( $\mathrm{F} \%=42,85 \% ; \mathrm{F}+\%=75,00 \%)$, Leonardo $(\mathrm{F} \%=41,66 \% ; \mathrm{F}+\%=100,00 \%)$ e Cristiano $(\mathrm{F} \%=56,25 \% ; \mathrm{F}+\%=66,66 \%)$ indicaram sobreinvestimento no estabelecimento das fronteiras. Especialmente em Leonardo, em que todas as respostas de forma estavam vinculadas a F+, o que, segundo Chabert (1998/2003), denota rigidez, pois a presença de F- é um indicador de permeabilidade do psiquismo diante de questões que emergem do inconsciente.

As respostas barreira-penetração nos protocolos de Cristiano (2:3), Paulo (2:2), Leonardo (5:3) e Jonas (4:3) encontram-se rebaixadas, indicando, portanto, a fragilidade das bases narcísicas, o que pode ser comum na adolescência (Jeammet, 2006). No entanto, quando essas respostas são acompanhadas de outros indicadores podem apontar para o comprometimento patológico do funcionamento psíquico. Nesse grupo, essa característica aponta para a utilização de defesas que dificultarão a emergência dos processos criativos em direção à elaboração dos conflitos psíquicos (Chabert, 1993; Emmanuelli \& Azoulay, 2008). Assim, é possível observar um movimento mais inibitório, principalmente com defesas relacionadas ao congelamento dos movimentos pulsionais (negação da fonte interna da pulsão, ausência de cinestesias libidinais ou agressivas, ausência de cor), em Jonas, Leonardo e Paulo. Por outro lado, em Cristiano as defesas de desdobramento (presença de representações de movimento especulares e respostas de reflexo) manifestam-se no protocolo.

\section{O investimento na representação de si, a identidade e a identificação}

A problemática identitária pode ser analisada pelas respostas de conteúdo humano no protocolo. As respostas $\mathrm{H}$ indicam a capacidade de identificação da pessoa em relação a uma imagem humana, habilidade que fundamenta a identidade (Chabert, 1998/2003; Emmanuelli \& Azoulay, 2008). Na adolescência, é esperado maior número de respostas $\mathrm{H}$ e $\mathrm{A}$ em relação às parcializações $\mathrm{Hd}$ e Ad (Jardim-Maran, 2011). Esse aspecto foi percebido nos protocolos de Cristiano (H+A:Hd+Ad=12:7), Paulo $(\mathrm{H}+\mathrm{A}: \mathrm{Hd}+\mathrm{Ad}=9: 2)$ e Leonardo $(\mathrm{H}+\mathrm{A}: \mathrm{Hd}+\mathrm{Ad}=8: 0)$; porém, a presença de respostas híbridas nos 4 testes analisados e o maior número de respostas $\mathrm{Hd}$ em relação a $\mathrm{H}$ no caso de Cristiano $(\mathrm{H}: \mathrm{Hd}=1: 5)$ podem indicar a presença de fragilidade identitária nesses adolescentes (Chabert, 1998/2003). Em Jonas (H+A: Hd+Ad=7:10), a problemática identitária é mais grave em função do número excessivo de conteúdos parciais e da ausência de $\mathrm{H}$ $(\mathrm{H}: \mathrm{Hd}=0: 6)$.

Ao se relacionar a dimensão identitária à identificação, uma análise mais detalhada das percepções humanas nas pranchas do Rorschach indica que também há variações no grupo. À exceção de Paulo, que apresenta uma proporção de $\mathrm{H}: \mathrm{Hd}=3: 1$, os demais adolescentes evidenciam restrição na capacidade de identificação e empatia com o humano. Isso se agrava particularmente nos protocolos de Leonardo e Jonas, que apresentam, respectivamente, proporções de $\mathrm{H}: \mathrm{Hd}=1: 0$ e 0:6.

No que se refere à imagem do corpo e à representação de si, a análise das respostas K e kan são especialmente relevantes. As respostas $\mathrm{K}$ revelam o humano em sua integridade; as kan podem expressar o conflito com mais facilidade, uma vez que estão deslocadas em conteúdos animais (Chabert, 2004). Na análise dos quatro protocolos, à exceção de Paulo, que apresenta duas cinestesias humanas, os demais se restringem a uma ou nenhuma resposta K. Jonas é quem apresenta uma problemática identitária mais grave, sem nenhuma resposta humana cinestésica no protocolo, e somente com representações parciais $(\mathrm{H}: \mathrm{Hd}=0: 6)$. Cristiano responde, na prancha VII: "duas pessoas com a cabeça encostando", e Leonardo, na prancha III, "duas pessoas trabalhando". Nos dois casos, a representação humana manifesta-se diluída e sem uma identificação sexual. No caso de Paulo, ele responde, na prancha III, "duas mulheres mexendo em alguma coisa ou dois homens" e, na prancha VII, "uma mulher se olhando no espelho". Há referência identificatória com o feminino em uma perspectiva narcísica e especular. 
Esse aspecto da identificação com o feminino, observado no caso de Paulo, está de acordo com a pesquisa de Roman (2004) para essa população de adolescentes.

A proporção entre as cinestesias maiores e menores revela aumento da segunda parte da fórmula, em que $\mathrm{K}<\Sigma \mathrm{k}$. Em todos os casos, a alta incidência das pequenas cinestesias, em detrimento da cinestesia humana inteira, denota dificuldade de integrar a imagem corporal em uma representação de si bem estabelecida, que permita realizar o manejo da pulsão e do afeto. Isso, associado ao predomínio de detalhes humanos parciais, compromete a constituição da identificação.

A análise da prancha $V$ é relevante para a compreensão das questões identitárias (Raush de Traubenberg, 1970/1998). Os protocolos dos adolescentes apresentam respostas banais sem qualificativos e respostas com conteúdo de estranheza, híbridas e de espelhamento (reflexo). Como exemplo, no caso de Jonas, a resposta dada a essa prancha é "um cachorro... Dois cachorros. Como esse desenho aqui é espelhado, o que tem de um lado, tem do outro". Como apontam Passalacqua e Gravenhorst (2005), as respostas de reflexo indicam problemática narcísica, em que o sujeito se relaciona com um outro que é o prolongamento de si mesmo, ou seja, um espelho. Nesse sentido, evidencia a problemática narcísica-identitária e o modelo da relação objetal, marcadamente estabelecido de forma especular.

\section{A dinâmica psíquica e algumas hipóteses sobre a passagem ao ato violento}

Os dados do protocolo de Cristiano indicam certa restrição da capacidade de adaptação e de compartilhamento do pensamento comum ( $\mathrm{Ban}=9,3 \%$ ), mas a relação com a realidade encontra-se preservada $(\mathrm{F} \%=56,2 \%$; kan na prancha VIII). Do ponto de vista emocional, sobressai o aspecto restritivo dessa dimensão: o seu tipo de vivência é coartativo ( $\mathrm{TV}=\Sigma \mathrm{K}: \Sigma \mathrm{C}=1: 1,5) \mathrm{com}$ tendência introversiva (Índice de Reatividade Afetiva IRA $=100 x V I I I+I X+X / R=31,20 \%) ;$ a capacidade de manejo do afeto é restrita $(\mathrm{FC}=1)$; o adolescente apresenta sinais de defesas rígidas e com a presença de afeto ansioso e depressivo $\left(\mathrm{F}+\%=66,6 \% ; \mathrm{FC}^{\prime}=2 ; \mathrm{FE}=3\right)$. O movimento pulsional primitivo, no entanto, está aumentado, indicando o rebaixamento do controle interno $(\mathrm{K}: \mathrm{kan}+\mathrm{kob}+\mathrm{kp}=1: 7)$; a rigidez das defesas e o controle dos impulsos reduzem as possibilidades de passagem ao ato $(\Sigma \mathrm{C}: \Sigma \mathrm{E}=0,5: 1,5)$. Por outro lado, a dificuldade de lidar com o afeto e os bloqueios tornam o adolescente vulnerável e reduzem a mobilidade psíquica, bem como a capacidade de adaptação às situações de grande demanda, exacerbando a passividade.

Os dados dos protocolos do Rorschach de Paulo e Leonardo, quando comparados aos indicadores das normas para adolescentes (Jardim-Maran, 2011; JardimMaran et al., 2015), revelam a inibição e o restrito manejo pulsional desses adolescentes. No caso de Paulo, o protocolo é restritivo $(\mathrm{R}=15)$, com busca de formalização do pensamento $(\mathrm{F} \%=53,33 ; \mathrm{F}+\%=37,50)$ e rebaixamento da responsividade afetiva $(\mathrm{FC}: \mathrm{CF}+\mathrm{C}=1: 0)$. No caso de Leonardo, a restrição do protocolo $(\mathrm{R}=12)$, o rebaixamento das respostas de forma $(\mathrm{F} \%=41,66)$, a baixa responsividade afetiva ( $\mathrm{FC}: \mathrm{CF}+\mathrm{C}=1: 1)$ e uma única resposta de movimento humano $(\mathrm{K}=1)$ apontam para a inibição. De maneira geral, essa inibição pode indicar restrição da capacidade psíquica de mentalização, na qual a passagem ao ato substitui o trabalho psíquico de ligação em um funcionamento com suporte narcísico identitário frágil, tal como na hipótese levantada por Marty $(2000,2010,2012)$. Essa hipótese também foi abordada por Amparo e Pereira (2010) na análise do protocolo de Rorschach de um adolescente que praticou ato sexual violento, indicando como o ato pode substituir a capacidade de pensar e lidar psiquicamente com emoções e afetos.

Já o protocolo de Jonas apresenta uma configuração diferenciada, denotando a reatividade afetiva e a sua ligação com o ambiente (VIII+IX+X/R=32,14\%), bem como a possibilidade de passagem ao ato por maior descontrole dos impulsos ( $\Sigma \mathrm{C}: \Sigma \mathrm{E}=7: 2 ; \mathrm{K}: \Sigma \mathrm{k} 0: 1)$. Diferentemente dos outros casos, esse adolescente revela outro modo de funcionamento psíquico, no qual a passagem ao ato pode ter uma função de defesa contra angústias muito cruas e primitivas e contra certa depressividade ansiosa subjacente $(\mathrm{Hd}+(\mathrm{Hd})+$ Anat $+\mathrm{Sg}+\mathrm{Fg}+\mathrm{Sex} \mathrm{x}$ 100/R=42,85\%; FC':C'F+C=2:0; FE:EF+E=4:0).

De modo geral, um aspecto importante a ser observado nos protocolos dos adolescentes que cometeram abuso sexual, e que demanda investigação posterior mais aprofundada, é a presença de indicadores da sensibilidade depressiva. Isso permite discutir a hipótese levantada por Chagnon $(2008,2009)$ e Marty $(2000,2010,2012)$ de que a passagem ao ato violento pode ser uma defesa contra a depressão. Nos protocolos dos adolescentes, essa sensibilidade depressiva e os sinais de ansiedade controlados pelo aspecto intelectual são identificados nos indicadores FC':C'F+C' e FE:EF+E, e podem ser caracterizados no comportamento pelos sinais de tédio. Emmanuelli e Azoulay (2008) relacionam essa questão da evocação da depressividade nos métodos projetivos com a sensibilidade à perda objetal e/ou narcísica na adolescência.

Em termos de conclusão, assim como nos casos analisados nesta pesquisa, outros estudos com adolescentes que passaram ao ato sexual violento apresentaram uma diversidade de funcionamentos psíquicos, sem a constituição de um perfil único (Chagnon, 2008, 2009, 2012; Roman, 2004, 2012). Nesse sentido, reafirma-se o que Jeammet (2006) argumentou acerca da importância de estudos que levem em conta aspectos econômicos e dinâmicos do funcionamento psíquico.

Dentre os aspectos do funcionamento psíquico analisados, os protocolos de Rorschach dos adolescentes indicaram vulnerabilidades no que se refere aos aspectos 
internos, particularmente aos fundamentos narcísicos e identitários. O estabelecimento consistente das bases narcísicas e identitárias possibilita suporte para o sentimento de continuidade de si e a segurança em relações objetais (Emmanuelli \& Azoulay, 2008; Jeammet \& Corcos, 2005). Quando essas bases falham e os afetos não podem ser elaborados, muitas vezes porque não se manifestam na vida psíquica, uma possibilidade de saída é o agir. A integração da agressividade e do afeto na vida psíquica requer fundamentos do narcisismo e da identidade bem constituídos.

As patologias do agir se configuram de forma independente do diagnóstico e de uma estrutura da personalidade. Nesse sentido, é necessário pensar para além do diagnóstico, nos fatores econômicos do psiquismo que abrangem essa problemática e na função de paraexcitação do psiquismo, ou seja, na sua capacidade de mentalizar e elaborar as angústias e os afetos (Jeammet, 2006).

No contexto da intervenção com essa população vulnerável, a avaliação psicológica é um processo que possibilita o acesso ao funcionamento psíquico, indicando fragilidades e pontos de apoio dos adolescentes para atravessar esse período de remanejamentos. Essa conclusão coloca a necessidade de a ação dos profissionais da Justiça e da Saúde incidir também sobre a subjetividade do adolescente e suas peculiaridades. É importante ressaltar, em relação ao adolescente que cometeu abuso sexual, além da intervenção jurídica, a necessidade de tratamento, cuidado e proteção. Sobre esse aspecto, Costa, Junqueira, Ribeiro e Meneses (2011) apontam para a necessidade de uma política de Saúde que tenha alcance também para esse grupo.

Em síntese, a infração de caráter sexual na adolescência relaciona-se de forma intrínseca com uma problemática desse período, o que torna inviável qualquer generalização. Este estudo com grupo único não teve como objetivo encontrar resultados generalizáveis. Considera-se, no entanto, que o Método de Rorschach permitiu apontar, nos casos estudados, uma problemática comum e com especificidades nas suas variações individuais. É importante, no entanto, realizar estudos com amostras mais abrangentes e que considerem as particularidades das condições de realização do abuso sexual, como, por exemplo, o abuso intrafamiliar e suas repercussões psíquicas tanto na vítima quanto no abusador.

Nesse sentido, este estudo aponta para a necessidade de realizar pesquisas futuras que considerem diferentes contextos de abuso sexual, bem como outros tipos de passagem ao ato. Essas possibilidades investigativas podem favorecer uma compreensão mais ampla sobre a violência e o funcionamento psíquico dos adolescentes.

\section{Referências}

Aberastury, A., \& Knobel, M. (1981). Adolescência. Porto Alegre: Artes Médicas. (Obra original publicada em 1970).

Amparo, D. M., \& Pereira, M. S. (2010). Adolescência e passagem ao ato violento: aspectos clínicos e psicodinâmicos. Em D. M. Amparo, S. F. C. Almeida, K. T. R. Brasil, \& F. Marty. (Eds.), Adolescência e violência: Teorias e práticas nos campos clínico, educacional e jurídico. (pp. 67-88). Brasília: Líber Livro.

Azoulay, C., Emmanuelli, M., Rausch de Traubenberg, N., Corroyer, D., Rozencwajg, P., \& Savina, Y. (2007). Les données normatives françaises du Rorschach à l'adolescence et chez le jeune adulte. Psychologie Clinique et Projective, 13(1), 371-409. doi: 10.3917/pcp.013.0371

Balier, C. (2008). Psychanalyse des comportements sexuels violents. (3a ed.). Paris: Presses Universitaires de France. (Obra original publicada em 1996).

Blos, P. (1998). Adolescência: Uma interpretação psicanalítica. (2a ed.). São Paulo: Martins Fontes (Obra original publicada em 1985).

Chabert, C. (1993). A psicopatologia no exame do Rorschach (N. da Silva Jr., Trad.). São Paulo: Casa do Psicólogo.

Chabert, C. (2003). O Rorschach na clínica do adulto. Lisboa: Climepsi Editores. (Obra original publicada em 1998).

Chabert, C. (2004). Psicanálise e métodos projetivos (A. José Lelé \& E. M. A. Costa e Silva, Trad.). São Paulo: Vetor.

Chagnon, J. Y. (2008). Traumatismo, violência e agressões sexuais. Em S. R. Pasian, E. T. K. C. Okino, S. R. Loreiro, \& F. L. Osório (Eds.), Avaliação da personalidade: Técnicas e contextos diversos: V Encontro da Associação Brasileira de Rorschach e Métodos Projetivos. (vol. 4, pp. 510-535). Ribeirão Preto: SBRo.

Chagnon, J. Y. (2009). A agressão sexual na adolescência: um destino da hiperatividade? Ágora: Estudos em Teoria Psicanalítica, 12(2), 275-290. doi: 10.1590/S1516-14982009000200008

Chagnon, J. Y. (2012). As agressões sexuais na adolescência. Em D. M. Amparo, S. F. C. Almeida, K. T. R. Brasil, M. I. G. Conceição, \& F. Marty (Eds.), Adolescência e violência: Intervenções e estudos clínicos, psicossociais e educacionais. (pp. 59-78). Brasília: Editora UnB.

Chagnon, J. Y. (2013). L'École de Paris: Bref historique. Le Carnet Psy, 169(2), 27-29. doi:10.3917/lcp.169.0027

Costa, L. F., Junqueira, E. L., Ribeiro, A., \& Meneses, F. F. F. (2011). "Ministério de Obrigação adverte": É preciso proteger os adolescentes ofensores sexuais. Avances en Psicologia Latinoamericana, 29(1), 33-46. Recuperado de https://dialnet.unirioja.es/servlet/ articulo? codigo $=3699940$

Durat Júnior, C. (2006). O Rorschach na avaliação psicológica do adolescente em conflito com a lei. (Dissertação de mestrado). Universidade São Francisco, Itatiba SP. Recuperado de http://livros01.livrosgratis.com.br/cp033351.pdf

Emmanuelli, M., \& Azoulay, C. (2008). As técnicas projetivas na adolescência: Uma abordagem psicanalítica (M. A. de Souza, Trad.). São Paulo: Vetor.

Gutton, P. (1990). Le pubertaire. Paris: PUF.

Gutton, P. (2002). Violence et adolescence. Paris: In Press Éditions. 
Husain, O. (1991) Sélection de l'échantillon en recherche projective: Pour une défense du groupe unique à faible visibilité groupale. Bulletin de Psychologie, 44(402), 465-468.

Ikiz, T. T., Zabci, N., Dusgor, B. P., Atak, I. E., Yavuz, E., Purisa, S., \& Catagy, P. (2010). Étude normative du Rorschach de la population adolescente turque. Psychologie Clinique et Projective, 16(1), 209-232. doi: 10.3917/pcp.016.0209

Jardim-Maran, M. L. S. (2011). O psicodiagnóstico de Rorschach em adolescentes: Normas e evidências de validade. (Tese de doutorado). Universidade de São Paulo, Ribeirão Preto S.P.

Jardim-Maran, M. L. S, Pasian, S. R., \& Okino, E. T. K. (2015). Normative study of Rorschach (Parisian School) for Brazilian adolescents. Paidéia, 25(62), 333-342. doi: 10.1590/1982-43272562201507

Jeammet, P., \& Corcos, M. (2005). Novas problemáticas da adolescência: Evolução e manejo da dependência. São Paulo: Casa do Psicólogo.

Jeammet, P. (2006). As patologias do agir na adolescência. Em M. Amaral (Ed.), Educação, psicanálise e direito. (pp. 22-56). São Paulo: Casa do Psicólogo.

Marty, F. (2000). Violences à l'adolescence. Em F. Marty (Ed.), L'illégitime violence: La violence et son dépassement à l'adolescence. (pp. 07-17). Ramonville Saint-Agne: Collection actualité de la psychanalyse.

Marty, F. (2010). Adolescência e violência. Em D. M. Amparo, S. F. C. Almeida, K. T. R. Brasil, \& F. Marty (Eds.), Adolescência e violência: Teorias e práticas nos campos clínico, educacional e jurídico. (pp. 45-66). Brasília: Editora UnB.

Marty, F. (2012). A função do agir na adolescência. Em D. M. Amparo, K. T. R. Brasil, S. F. Almeida, M. I. Conceição \& F. Marty (Eds.). Adolescência e violência: Intervenções e estudos clínicos, psicossociais e educacionais. (pp. 19-31). Brasília: Editora UnB.

Masling, J. (1999). Bodies under investigation: A review of Body Image and Personality by S. Fisher and S.E. Cleveland (1958). Journal of Personality Assessment, 72(1), 164-174.

Pasian, S. R. (2010). O psicodiagnóstico de Rorschach em adultos: Atlas, normas e reflexões. São Paulo: Casa do Psicólogo.

Passalacqua, A. M., \& Gravenhorst, M. C. (2005). Os fenômenos especiais no Rorschach. São Paulo: Vetor Editora.

Pelladeau, E., \& Chagnon, J. Y. (2015) Modalités identificatoires et dérives narcissiques dans les actes d'agressions sexuelles à l'adolescence: Considérations à partir d'un cas. Psychologie Clinique et Projective, 21(1), 63-91.

Rausch de Traubenberg, N. R., \& Sanglade, A. (1984). Représentation de soi et relation d'objet au Rorschach grille de représentation de soi. Revue de Psychologie Appliquée, 34(1), 41-59.

Rausch de Traubenberg N. (1998). A prática do Rorschach (Tradução A. J. Lelé). São Paulo: Vetor. (Original publicado em 1970). Recuperado de http://www.scielo.br/scielo.php?script=sci_nlinks\&pid=S0103-863X201500030033300000\&lng=en

Roman, P. (2004). La violence sexuelle et le processus adolescent. Dynamique des aménagements psychiques. des auteurs aux victimes de violence sexuelee. L'apport des méthodes projectives. Revue de la Société du Rorschach et des méthodes projectives de langue Françoise, 10(1), 113-146. Recuperado de https://www.cairn.info/article.php?ID_ARTICLE=PCP_010_0113

Roman, P. (2012). Les violences sexuelles à l'adolescence: Comprendre, accueillir, prévenir. Issy-lès-Moulineaux: Elsevier Masson.

Souza, C. C., \& Resende, A. C. (2012) Transtornos psicológicos em adolescentes socioeducandos. Avaliação Psicológica, 11(1), 95-109. Recuperado de http://pepsic.bvsalud.org/pdf/avp/v11n1/v11n1a10.pdf

Verdon, B., \& Amparo, D. M. (2013). Entre a atividade perceptiva e a fantasmática: Nina Rausch de Traubenberg e o método de Rorschach. Boletim Academia Paulista de Psicologia, 33(85), 230-242. Recuperado de http://www.redalyc.org/articulo.oa?id=94629531002

Winnicott, D. W. (2005). Privação e delinquência (4a ed., A. Cabral, Trad.). São Paulo: Martins Fontes (Obra original publicada em 1984).

\section{Sobre os autores}

Lana dos Santos Wolff é Psicóloga da Secretaria de Saúde do Distrito Federal, Doutoranda em Psicologia no Programa de Psicologia Clínica e Cultura da Universidade de Brasília.

Deise Matos do Amparo é Professora do Programa de Psicologia Clínica e Cultura da Universidade de Brasília, Doutora em Psicologia, Pós-Doutorado pela Université Paris V, Pesquisadora Bolsista Produtividade Nível 2 Conselho Nacional de Desenvolvimento Científico e Tecnológico.

Roberto Menezes de Oliveira é Professor da Universidade Católica de Brasília e Doutor em Psicologia pela Universidade de Brasília.

Jean Yves Chagnon é Psicólogo, Psicanalista e Professor da Université Paris 13- França, e Membro do Laboratório Unité Transversale de Recherche Psychogenèse et Psychopathologie. 